\title{
A Review on Analysis of Heat Transfer Coefficient in Internal Rib Tube
}

\author{
Rahul Kumar \\ Research Scholar \\ M. Tech. \\ Technocrats Institute of Technology \& Science \\ Bhopal (M.P), India \\ rahulxp151@.gmail.com
}

\author{
Dr Sukul Lomash \\ Associate Professor \\ Technocrats Institute of Technology \& Science \\ Bhopal (M.P), India \\ lomash15@gmail.com
}

\begin{abstract}
The rate of heat transfer is increased by a fin arrangement applied to the rectangular duct. In order to save energy, many industrial heat exchange devices use various techniques to improve heat transfer, such as: $B$. fins, dimples, vortex chamber and ribbed tabs. The most common technique used to improve heat transfer is to add fins to the flow channel. The ribs are of simple construction and are widely used in many industries. Turbulent kinetic energy generation increases turbulent heat transfer in the duct due to flow disturbances caused by fin arrangements. The shape of the fin plays an important role in improving heat transfer as it affects the formation of bubble separation behind the fin and the amount of turbulent kinetic energy generated.
\end{abstract}

Keywords: heat exchanger, rib tube, heat transfer, temperature.

\section{INTRODUCTION}

Ribs are a popular heat transfer enhancement device used in various heat exchange channels such as internal cooling channels in gas turbine blades. The disturbance of the flow caused by the arrangements of the fins greatly increases the generation of turbulent kinetic energy, which improves the turbulent heat transfer in the channel. Among the many geometric parameters related to the positioning and configuration of the ribs, the shape of the cross section of the rib influences the formation of a separating bubble behind the rib and the amount of turbulent kinetic energy generated; therefore, the shape of the fin is an important factor that determines the heat transfer performance of the fin.
The heat transfer from surface may in general be enhanced by increasing the heat transfer coefficient between a surface and its surrounding or by increasing heat transfer area of the surface or by both. Ribs used in cooling channel and heat exchanger channel are most commonly used passive heat transfer techniques. So that the work related to fluid flow and heat transfer in ribbed channel is go so far. This heat transfer augmentation technique are applied to many industrial application such as shell and tube type heat exchanger , electronic cooling devices, thermal regenerators, and internal cooling system of gas turbine. Each rib on downstream separates the flow, recirculate, and impinges on channel wall and these are the main reason for heat transfer enhancement in such channel. The use of rib in heat exchanger not only increase the heat transfer rate but also substantial the pressure loss [1]. The rib arrangement and geometry resulting in different heat transfer distribution by altering the flow field. Therefore by making the modification in rib geometry we can increase the heat transfer rate but at the same time we need to consider the pressure drop also because it increases significantly.

The study of improved heat transfer performance is referred to as heat transfer augmentation, enhancement. The common thermo hydraulic goals are to reduce the size of a heat exchanger required for a specified heat duty and to upgrade the capacity of an existing heat exchanger, also to reduce the pumping power. Heat transfer enhancement techniques are commonly used in areas such as process industries heating and cooling in evaporators, thermal power plants, air conditioning equipment's, refrigerators and radiators for space vehicles, automobiles. 
These are classified into three categories

\section{- Passive techniques}

- Active techniques

In passive techniques inserts are used in flow passage to augment the heat transfer rate and it is advantageous compared with active techniques because the insert manufacturing process is simple and these techniques are easily employed in existing heat exchanger. For design of compact heat exchanger passive techniques of heat transfer augmentation can plays the important role if proper insert configuration can be selected according to the heat exchanger working condition. Several studies are taken on passive techniques of heat transfer augmentation. Most commonly used passive heat transfer augmentation techniques are ribs twisted tape, wire coils, fins dimples etc. in active heat transfer enhancement techniques external power is required to cause the desired flow modification and improvement in the rate of heat transfer [2]. These methods are not commonly used because of need of external power. Compound techniques are the combination of passive and active heat transfer enhancement techniques.

Higher inlet temperatures are required for high turbine efficiency. Hot gas path turbine components are subjected to elevated heat loads from hot gases exiting the combustor section. To protect the hot gas path components from hot gases, sophisticated internal and external cooling concepts are employed in turbine airfoils. Relatively colder air bled off from the compressor section is routed through complicated internal cooling passages to cool the hot internal walls of turbine airfoils. However, the coolant bled from the compressor reduces the turbine efficiency. Hence, it is imperative to develop cooling concepts which have high heat transfer enhancement levels at relatively lower enhancement in pumping power. Considerable research has been carried out to develop internal cooling concepts that have high rates of heat transfer between the coolant and the blade internal walls.
Aadel A. R. Al Kumait et al. [3] The study is accomplished by using the finite volume method, and its objective involves finding a low friction factor and high heat transfer enhancement in the presence of TiO 2/water nanofluids. In comparison with the plain tube, a helical ribbed tube provides higher performance evaluation criteria (about $2.0 \%$ ), while circumferentially ribbed tube provides $1.9 \%$ and longitudinal ribbed tube provides $1.88 \%$. The helical ribbed tubes with a $5.89 \mathrm{~mm}$ pitch distance gave higher turbulent kinetic energy due to a stronger swirl intensity, resulting in a thinner thermal boundary layer and a higher Nusselt number with uniform distribution.

Zhanwei Liu et al. [4] In this study, single-phase heat transfer enhancement in internally finned tubes is investigated numerically. The influence of fin number, helix angle, fin height, fin width, and shape on the flow and heat transfer characteristics is studied. The research results indicate that the resistance coefficient and Nusselt number both increase with the increment of these parameters, among which the helix angle has the largest impact on the heat transfer enhancement.

Slawomir gradziel et al. [5] This paper presents the experimental determination of the coefficient in an internally rifled tube with spiral ribs. The tests are carried out on a laboratory stand constructed at the Institute of Thermal Power Engineering of the Cracow University of Technology. Comparing the Nusselt number values calculated based on the developed correlation with those obtained using other correlations described in the literature, it can be observed that the criterial number is about twice higher. The research results confirm the thesis that the element internal geometry has a substantial impact on the heat transfer process.

Kute S. B et al. [6] Computational fluid dynamics were used to simulate and study the heat transfer of both plain tube and helically ribbed tube for fire tube boiler. The surface temperature distribution of plain tube and rifled tube is presented in this paper. The result indicates the helically ribbed tube has higher heat transfer rate than plain tube.

\section{LITERATURE REVIEW}

\section{A. RIB-GROOVE ARRANGEMENT}


Mi-Ae Moon, Mean-Jung Park [7] the heat transfer and frictional power dissipation of a roughened rectangular duct with various cross sections were analyzed using a three-dimensional Reynold mean Navier-Hub equation. They performed experiments on 16 shapes of ribs which are house-shaped, trapezoidal, trapezoidal, bootshaped, boot-shaped, etc. They examined the influence of the Reynolds number and the ratio of rib spacing to width on the performance of various ribs for the Reynolds number 5,000 - 50,000 and the ratio of the rib width to pitch of $5.0-10,0$. They reported that the new boatshaped fin design showed the best heat transfer performance compared to the square fin with medium friction power dissipation.

Monsak Pimsarn, Pongjet Promvonge [8] the experiments were performed on a Z-shaped fin with a Zshaped fin placed on the rectangular duct at $30^{\circ}, 45^{\circ}$ and $60^{\circ}$ with respect to the direction of the air flow. Experiment on a rectangular duct with aspect ratio $A R=$ 10 , height $\mathrm{H}=30 \mathrm{~mm}$ with rib height in $\mathrm{Z}$ and $/ \mathrm{H}=0.2$ and rib spacing $(P), P / H=3$. The results show that 45 - $Z$ ribs reduce heat transfer rate compared to smooth channel, flat rib, $30^{\circ}$ and $60^{\circ} \mathrm{Z}$ ribs with $\mathrm{e} / \mathrm{H}=0.2$ and $\mathrm{P} / \mathrm{H}=3$ to $\operatorname{Re}=5000$ to 25000 . The factor thermal improvement for the $Z$ ribs is 150 to $160 \%$ compared to the flat ribs and $225-255 \%$ for the smooth channel.

R. Tauscher, F. Mayinger [9] deals with the experimental and numerical study of thermal transfer by forced convection in flat channels with rectangular section. It uses a Reynolds number between 500 and 10000 . They looked at various configurations such as the shape of the ribs, size, spacing, angle of attack, layout, width and height of the channel. They evaluated the average heat transfer and heat transfer performance by measuring the average liquid temperature at the inlet and outlet of the test section. They reported that the ratio of rib spacing to height was more effective than $\mathrm{P} / \mathrm{e}$ $=10$ and adding a groove to the rib spacing had better results.

Smith Eiamsa- ard, Pongjet Promvonge [10] were experimentally performed to examine the combined effect of ribbed ribbed fins on heat transfer by turbulent forced convection in a rectangular duct. They use a three-rib groove arrangement, rectangular rib and triangular groove, triangular rib with rectangular groove and triangular rib with triangular groove with aspect ratio $A R=$ 10.24113/ijoscience.v6i12.355
20 , channel height $\mathrm{H}=9 \mathrm{~mm}, \mathrm{e}=3 \mathrm{~mm}$ with a ratio of three Pich PR $=6.6,10,13.3$.

The result showed that rectangular fins with a triangular groove arrangement provide the highest heat transfer rate and the highest friction factor compared to others. While a triangular rib with a triangular groove provides a higher thermal improvement index for all pitch ratios. The ribbed tabs at $\mathrm{PR}=6.6$ offer up to $80 \%$ superior heat transfer. The thermal improvement index is higher when using a tab with a lower Reynolds number.

\section{RiB Geometry}

Large number of Rib geometries has been proposed for the use of heat transfer enhancement and more are still being developed

\section{A. Common Attributes of Ribs are}

a) Shape: Most of the Ribs found in literature are Rectangular Ribs, Square Ribs, Triangular Ribs, M-shaped, W-shaped, VShaped. In the present work, W-shaped ribs have been studied.

b) Height: Small heights of ribs are preferred to minimize the pressure drop.

c) Spacing: It is the distance between two consecutive Ribs.

d) Width: Small widths of ribs are preferred to minimize the pressure drop.

\section{ARRANGEMENTS OF RIBS}

Arrangement of Ribs on a flat plate plays a crucial role to enhance the heat transfer within the channel without incurring the penalties of friction and pressure drop that are severe enough to negate the benefits of heat transfer augmentation. Inline, Staggered, Offset, Zig-Zag are the possible arrangement of Ribs in a flow channel.

\section{TYPES OF RIBS}

Implementation of Ribs are decided on the basis of size, cost and their ability to lend support to the tube bundles and direct flow, often this is linked to available pressure 
drop and the size and number of passes within the exchanger. Special allowance changes are made for finned tubes. The different types of Ribs are:

\section{- V-Shaped ribs}

- M-shaped ribs

- W-shaped ribs

- Circular ribs

- Rectangular ribs

\section{Conclusion}

The study of heat transfer performance improvement is called heat transfer improvement and improvement. Common thermo hydraulic goals are to reduce the size of a heat exchanger needed for a given heat output and improve the capacity of an existing heat exchanger and reduce pumping capacity. Many researchers are interested in improving the rate of heat transfer using passive methods such as large areas, pits and rough surfaces. Review of the literature shows that heat exchanger fins have a wide range of uses. The rate of heat transfer is increased by using a groove spaced apart from the fin as the degree of turbulence increases. And the friction factor also increases. The choice of the split ratio and the geometry of the fins is very important because it affects the variation of the heat transfer with the Reynolds number and the friction factor.

\section{REFERENCES}

1. Miss. Ashwini Vasant Thakare, Dr. J. A. Hole "Experimental Validation of Heat Transfer Enhancement in plate Heat Exchanger with Non-Conventional Shapes of Rib" International Journal of Science, Engineering and Technology Research (IJSETR), Volume 5, Issue 3, March 2016.

2. Miss. Ashwini Vasant Thakare, Dr. Hole J. A. "Review of Heat Transfer Enhancement in Plate Heat Exchanger with Non-Conventional Shapes of Rib" International Journal of Engineering Research \& Technology (IJERT) ISSN: 2278-0181 Vol. 4 Issue 02, February-2015

3. Aadel A. R. Al Kumait Thamir K. Ibrahim "Experimental and numerical study of forced convection heat transfer in different internally ribbed tubes configuration using $\mathrm{TiO} 2$ nanofluid" Volume48, Issue5 05 April 2019

4. Zhanwei Liu, Yanwei Yue "Numerical Analysis of Turbulent Flow and Heat Transfer in Internally Finned Tubes" Energy Res., 18 July 2019.
5. Slawomir gradziel, Karol Majewski "experimental determination of the heat transfer coefficient in internally rifled tubes", Gradziel, S., et al.: Experimental Determination of the Heat Transfer Coefficient. thermal SCIENCE: Year 2019, Vol. 23, Suppl. 4, pp. S1163-S1174.

6. Kute S. B, Sonage B. K "Study of Surface Temperature Distribution of Plain tube and Rifled tube by CFD Analysis" International Research Journal of Engineering and Technology (IRJET), Volume: 05 Issue: 02 | Feb-2018.

7. Mi-Ae Moon, Min-Jung Park, Kwang-Yong Kim, Evaluation of heat transfer performances of various rib shapes, International Journal of Heat and Mass Transfer , pp. 275284, Jan 2014.

8. Monsak Pimsarn, Parkpoom Sriromreun, and Pongjet Promvonge, Augmented Heat Transfer in Rectangular Duct with Angled Z-Shaped Ribs, International Conference on Energy and Sustainable Development: Issues and Strategies, 2010.

9. R. Tauscher, F. Mayinger, Heat transfer enhancement in a plate heat exchanger with rib- roughened surfaces, SAE journal, pp. 207-221, 2009.

10. Smith Eiamsa-ard, Thermal characteristics of turbulent rib-grooved channel flows, International Communications in Heat and Mass Transfer, pp. 705711, May 2009. 